\title{
Comparison Two of Different Technologies for Outdoor Positioning of Robotic Vehicles
}

\author{
Miroslav Dvorak and Petr Dolezel \\ Faculty of Electrical Engineering and Informatics, \\ University of Pardubice, Czech Republic \\ \{miroslav.dvorak, petr.dolezel\}@upce.cz \\ http://www .upce.cz/
}

\begin{abstract}
This paper aims to compare two different technologies, which can determine the exact position of a robotic vehicle. The first method uses wireless technology and is based on the measurement of the signal strength of the bluetooth beacons. Based on these values, you can calculate the distance from beacons. The second method uses laser light and measurement of the reflected pulses. Based on the reference points of reflection, we can determine the distance. Both methods then use 2D triangulation to determine the position of the robotic vehicle. The exact position of the bluetooth beacons or the reference points must be known for the calculation. The paper also describes experiments with a laser, and the conclusion provides an evaluation of both technologies.
\end{abstract}

Keywords: bluetooth, iBeacon, laser, LiDAR, photodiode, RSSI, trilateration

\section{Introduction}

The requirements for the exact positioning of robotic vehicles are constantly increasing. There are various outdoor positioning technologies such as GPS [1], GALILEO [2] or GLONASS [3]. However, this article deals with the local determination of the position of a robotic vehicle. Examples include drawing lines on a football field or a parking places in front of supermarkets. In addition, the article does not look for a solution for a single robotic vehicle, but for a group of robotic vehicles. Therefore, it solves not only the method of mutual cooperation but also the detection of mutual collisions of vehicles and collisions of vehicles with obstacles, whereas the main goal is to develop a group of vehicles capable to draw patterns on an outdoor surface.

For this purpose, bluetooth wireless technology was considered as a first approach for robot positioning. As a reference point for determining distances, a device called iBeacon[4] was used. These devices periodically transmit their ID. Based on measured signal strength, you can estimate the distance from beacons. Using the $2 \mathrm{D}$ triangulation method, the position of the robotic vehicle was calculated. The second considered technology uses a laser beam to measure distance. A laser device sends out a short light pulse that is reflected from the 
measured object. The reflected pulse comes back and is captured by the optics of the device. The time, that the laser beam takes to complete the distance, is then calculated.

\section{2 iBeacon}

The iBeacon is a small and low-power device which periodically transmits its UUID (Universally Unique Identifier) number through the interface of Bluetooth version 4.x. Positioning using an iBeacon is described and tested in the article "An IoT Approach to Positioning of a Robotic Vehicle" [5]. In this article, theory about positioning using bluetooth technology is summarized. Hence, the robot vehicle measures signal strength of iBeacons, called RSSI (Received Signal Strength Indicator) [6], and the distance is calculated according to the strength. Based on these distances, a vehicle is able to estimate its position. According to the experiments presented in the cited paper, the overall accuracy of the position of the robotic vehicle is approximately $0.2 \mathrm{~m}$. The mentioned precision of in the position estimation of the robotic vehicle is, however, unacceptable. Therefore, this method is not suitable for further implementations.

\section{LiDAR}

The use of LiDAR (Light Detection And Ranging) for 2D localization seems more advantageous. In addition to the more precise position of the mobile vehicle, we can obtain the information needed to detect collisions of individual robotic vehicles. For experiments, the LiDAR lite v3 [7] is used. STM32 microcontroller is utilized as a control unit, and LiDAR is connected via the I2C bus.

Since LiDAR applies a method that measures the distance to a target by illuminating the target with pulsed laser light, while measuring the reflected pulses with a sensor, the robotic vehicle needs to be handled with the secondary continuous laser. This laser is used to focus on the target's active reference points. The short flashes of LiDAR are not capable of being detected by photodiodes used as the active reference points.

The mechanical structure consists of a gearbox and an optical sensor engine, and also two lasers attached to the transmission output shaft of the gearbox, as shown in Fig. 1. Information from the optical sensor is used to determine the angle of rotation of the lasers. This information, along with obstacle distance information, is used for collision detection.

\section{Active reference points of reflection}

The basis for the orientation of the robotic vehicle with laser is the active reference points. Each reference point consists a photodiode matrix with a filter to eliminate sunlight and a control unit, as shown in Fig. 2. 


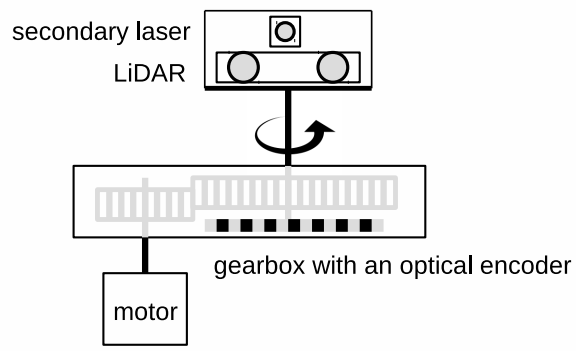

Fig. 1. Part of mechanical structure of robotic vehicle.

When a photodiode is illuminated by the secondary laser beam, the control unit sends the information containing a mark of the reference point and the positions of the photodiode. This information is sent as a UDP (User Datagram Protocol) broadcast using the WiFi interface. then, this information is received by all robotic vehicles. Nevertheless, only one vehicle uses this information for the calculation of the current position.

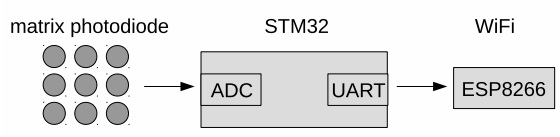

Fig. 2. Block diagram of active point reference.

The method of controlling robotic vehicles is based on an agent-oriented approach, so the agent periodically sends a request to find a position of a particular vehicle. The already mentioned WiFi interface is used for communication. This vehicle gradually focuses on the available reference points, and calculates its position using $2 \mathrm{D}$ triangulation [8] from the obtained responses.

\section{Mutual cooperation of group of robotic vehicles}

Common rendering of a pattern on an outdoor surface going to be based on mutual collaboration of group of robotic vehicles. As a considered solution of this problem, each robotic vehicle is fitted with a drawing unit and draws some parts of the pattern.

The required pattern is entered into the main application and the application divides it into individual curves, which are sequentially assigned to the nearest robotic vehicles. Therefore, the emphasis is placed on greater accuracy of positioning of individual vehicles. In the case of large inaccuracies, the curves would 
not accurately correspond with each other and the resulting shape would be unusable, as shown in Fig. 3.

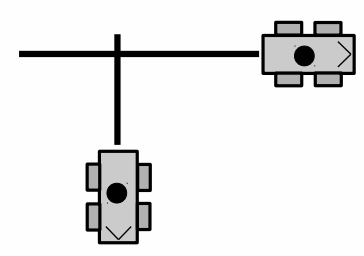

Fig. 3. Two robotic vehicles drawing letter T, incorrect positioning.

\section{Obstacle detection and collision of robotic vehicle}

Since LiDAR device detects not only the reference points, but also other objects, obstacle detection procedure can be implemented into the controlling system.

Each robotic vehicle, after receiving a command to move to a new position, begins to monitor its surroundings. As soon as it detects obstacles at a defined minimum distance, the vehicle automatically stops. The agent-oriented system [9] periodically asks for the status of robotic vehicles. Once it receives information about a possible collision, it sends a robotic vehicle command to explore the surroundings in more detail.
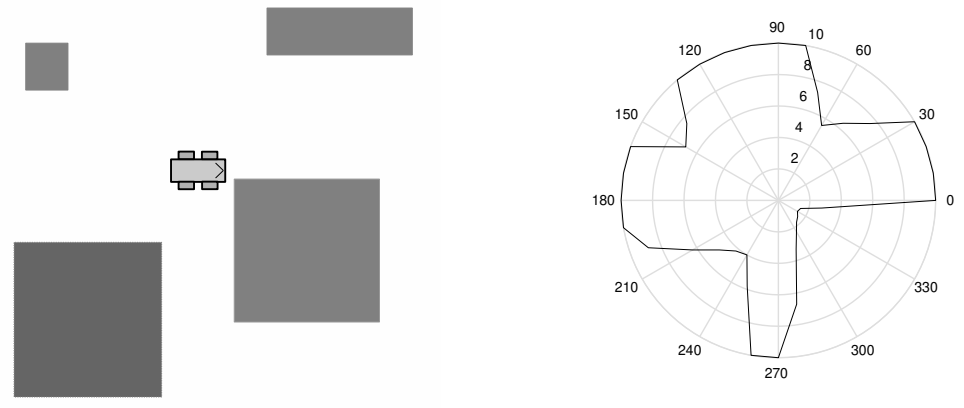

Fig. 4. Environment map

Based on these data, an agent-based system creates an environment map, as shown in Fig. 4. The system then evaluates whether it is a fixed obstacle or collision with another robotic vehicle. According to this, the system suggests another route or waits for the second moving vehicle to leave. 


\section{Experiments with LiDAR}

As the main contribution of this paper, a set of experiments is presented here in order to test the suitability of the use of LiDAR for precise determination of a robotic vehicle position.

\subsection{Measurement accuracy}

This experiment is focused on detecting the accuracy of LiDAR measurements. A precision of about $10 \mathrm{~cm}$ can be achieved according to the catalog sheet. Data acquisition is performed within a 1-10m range of distances with an increment of $1 \mathrm{~m}$. For each distance, 1000 values are obtained. The results of these measurements are shown in Table 1.

Table 1. Measurement results for individual distances.

\begin{tabular}{|c|c|c|c|}
\hline \multirow{2}{*}{ Real distance $(\mathrm{m})$} & \multicolumn{3}{|c|}{ Error (m) } \\
\cline { 2 - 4 } & $\min$ & $\max$ & average \\
\hline 1 & 0.0300 & 0.1200 & 0.0641 \\
\hline 2 & 0.0400 & 0.0800 & 0.0673 \\
\hline 3 & 0.0600 & 0.0900 & 0.0724 \\
\hline 4 & 0.1100 & 0.1500 & 0.1313 \\
\hline 5 & 0.1500 & 0.1900 & 0.1763 \\
\hline 6 & 0.1600 & 0.2000 & 0.1819 \\
\hline 7 & 0.1500 & 0.2000 & 0.1790 \\
\hline 8 & 0.1700 & 0.2100 & 0.1888 \\
\hline 9 & 0.1800 & 0.2200 & 0.1965 \\
\hline 10 & 0.1800 & 0.2400 & 0.2083 \\
\hline
\end{tabular}

\subsection{The effect of the angle of rotation of the reflection plate on the distance}

This experiment tests the influence of the rotation angle of the reference point reflector plate on the accuracy. The reflection plate is rotated in the range of $0^{\circ}$ $80^{\circ}$ with steps of $20^{\circ}$. This measurement was repeated for different distances, see Table 2.

It is clear from the measured values that the accuracy of the measurement depends very much on the angle of rotation of the reflection plate. A black reflector plate was used in this experiment. With the increasing distance from the reflection plate and increasing angle of the reflection plate, the distance values tend to be undetectable. The conclusion of this experiment demonstrates the inappropriate use of flat reflection surfaces for reference points. Thus, circular reflection surfaces are used for further experiments. 
Table 2. Influence of the angle of rotation of the reference point reflector plate

\begin{tabular}{|c|c|c|c|c|}
\hline \multirow{2}{*}{ Distance $(\mathrm{m})$} & \multirow{2}{*}{ Angle of rotation $\left(^{\circ}\right)$} & \multicolumn{3}{|c|}{ Error $(\mathrm{m})$} \\
\hline & & Min & Max & Average \\
\hline \multirow{5}{*}{1} & 0 & 0.0300 & 0.1200 & 0.0641 \\
\hline & 20 & 0.0900 & 0.1700 & 0.1300 \\
\hline & 40 & 0.1000 & 0.2200 & 0.1513 \\
\hline & 60 & 0.1300 & 0.2500 & 0.1937 \\
\hline & 80 & 0.1000 & 0.3300 & 0.2281 \\
\hline \multirow{5}{*}{2} & 0 & 0.0400 & 0.0800 & 0.0673 \\
\hline & 20 & 0.0900 & 0.2100 & 0.1213 \\
\hline & 40 & 0.1200 & 0.2500 & 0.1436 \\
\hline & 60 & 0.1400 & 0.2200 & 0.1540 \\
\hline & 80 & 0.1300 & 0.3600 & 0.2257 \\
\hline \multirow{5}{*}{3} & 0 & 0.0600 & 0.0900 & 0.0724 \\
\hline & 20 & 0.1000 & 0.1900 & 0.1125 \\
\hline & 40 & 0.1300 & 0.2700 & 0.1394 \\
\hline & 60 & 0.1400 & 0.2900 & 0.1527 \\
\hline & 80 & 0.1600 & 0.3200 & 0.1636 \\
\hline \multirow{5}{*}{4} & 0 & 0.1100 & 0.1500 & 0.1313 \\
\hline & 20 & 0.1500 & 0.2400 & 0.1507 \\
\hline & 40 & 0.1700 & 0.3200 & 0.2204 \\
\hline & 60 & 0.2300 & 0.4500 & 0.2657 \\
\hline & 80 & - & - & - \\
\hline \multirow{5}{*}{5} & 0 & 0.1500 & 0.1900 & 0.1763 \\
\hline & 20 & 0.1600 & 0.3400 & 0.1833 \\
\hline & 40 & 0.1800 & 0.3800 & 0.2256 \\
\hline & 60 & 0.2200 & 0.4800 & 0.3257 \\
\hline & 80 & - & - & - \\
\hline
\end{tabular}


Changing the shape of the reference point also results in a change in the design of the optical sensor.

The photodiodes are not placed in the matrix, but in individual layers consisting of disks of clear plexiglass, as showed in Fig. 5.

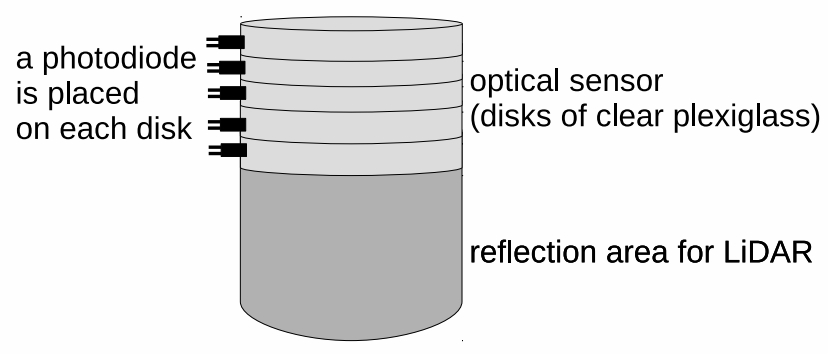

Fig. 5. Construction of circular reference points.

\subsection{D trilateration}

This experiment verifies the accuracy of robotic vehicle positioning using 4 reference points located in the corners of the test surface. The test surface sizes are set to $10 \mathrm{~m} \times 10 \mathrm{~m}$. The robot vehicle is placed in a known position, the measurement situation is shown in Fig. 6. For the comparison of both technologies, identical measurements were used to test iBeacons, as presented in [5]. The results of this measurement are shown in Fig. 7 and in Fig. 8. Apparently, the measurement using LiDAR is more accurate.

\section{Conclusion}

The main aim of the article is to compare two different technologies for robot positioning and to determine the more accurate one. For comparison, it is also necessary to take into account the complexity and cost of each individual solution.

Bluetooth technology is less accurate, but the cost of solutions is very low. The iBeacon is commonly available, and the robotic vehicle can be easily equipped with a conventional bluetooth module.

The solution using LiDAR is more accurate, but the circular reference points are not only more expensive, but also more demanding in terms of construction. This is, however, balanced by more precise positioning capability and the ability to use it as the obstacle detection component. 


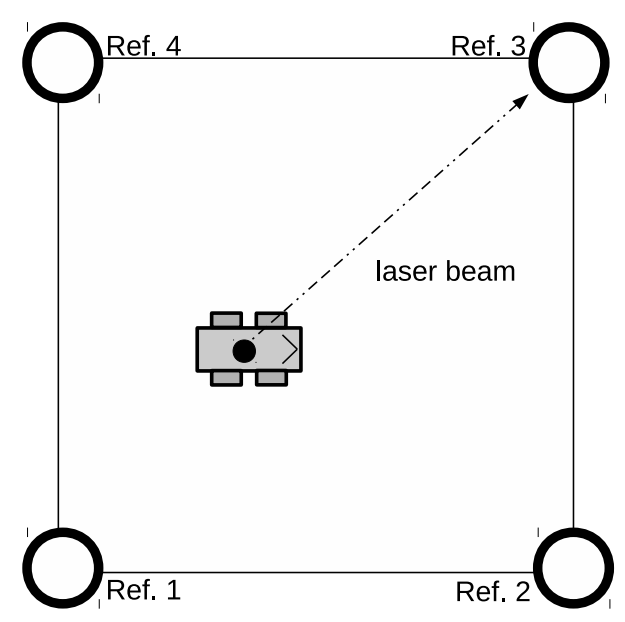

Fig. 6. Layout of experiment using LiDAR.

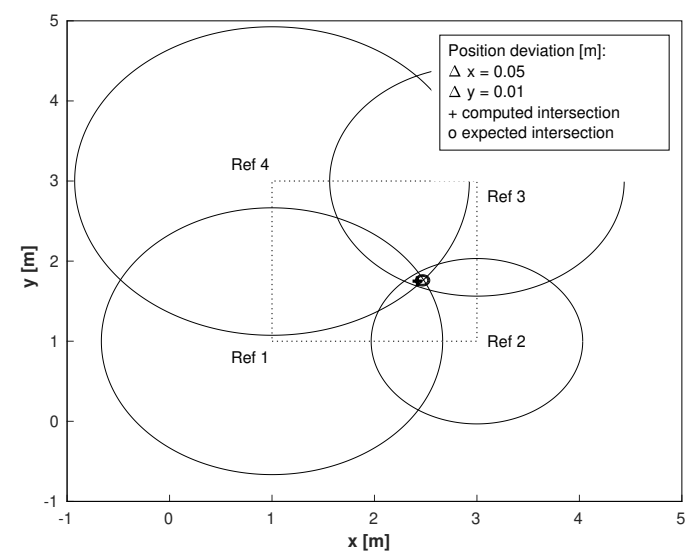

Fig. 7. Experiment using LiDAR. 


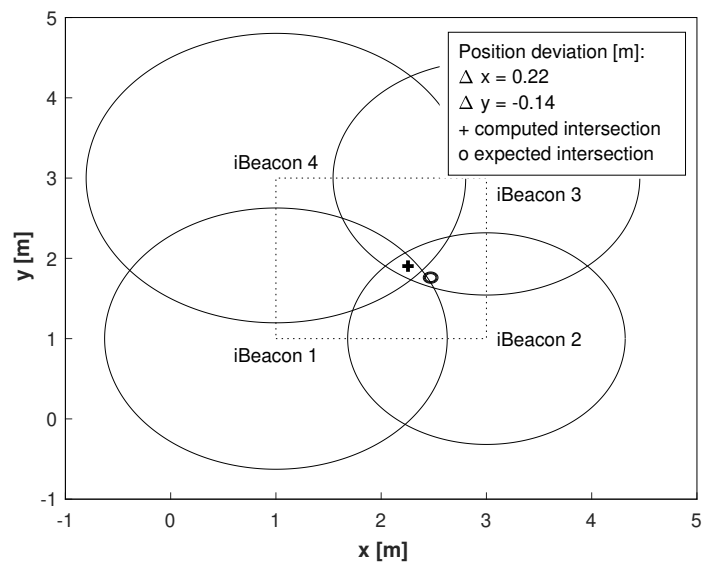

Fig. 8. Experiment using bluetooth technology.

\section{Acknowledgment}

The work has been supported by the Funds of University of Pardubice, Czech Republic. This support is very gratefully acknowledged.

\section{References}

1. A. Mulla, J. Baviskar, A. Baviskar and A. Bhovad, "GPS assisted Standard Positioning Service for navigation and tracking: Review \& implementation," 2015 International Conference on Pervasive Computing (ICPC), Pune, 2015, pp. 1-6. doi: 10.1109/PERVASIVE.2015.7087165

2. D. L. Kwasniak, "Single Point Positioning Using GPS, Galileo and BeiDou System," 2018 Baltic Geodetic Congress (BGC Geomatics), Olsztyn, 2018, pp. 310-315. doi: 10.1109/BGC-Geomatics.2018.00065

3. D. Kwaniak, S. Cellmer and K. Nowel, "Precise positioning using the modified ambiguity function approach with combination of GPS and Galileo observaions," 2018 25th Saint Petersburg International Conference on Integrated Navigation Systems (ICINS), St. Petersburg, 2018, pp. 1-6. doi: 10.23919/ICINS.2018.8405841

4. X. Li, D. Xu, X. Wang and R. Muhammad, "Design and implementation of indoor positioning system based on iBeacon," 2016 International Conference on Audio, Language and Image Processing (ICALIP), Shanghai, 2016, pp. 126-130. doi: 10.1109/ICALIP.201trilateration6.7846648

5. Dvorak M., Dolezel P. (2019) An IoT Approach to Positioning of a Robotic Vehicle. In: Silhavy R. (eds) Software Engineering and Algorithms in Intelligent Systems. CSOC2018 2018. Advances in Intelligent Systems and Computing, vol 763. Springer, Cham

6. R. K. Mahapatra and N. S. V. Shet, "Experimental analysis of RSSI-based distance estimation for wireless sensor networks," 2016 IEEE Distributed Computing, VLSI, Electrical Circuits and Robotics (DISCOVER), Mangalore, 2016, pp. 211-215. doi: 10.1109/DISCOVER.2016.7806221 
7. A. N. Catapang and M. Ramos, "Obstacle detection using a 2D LIDAR system for an Autonomous Vehicle," 2016 6th IEEE International Conference on Control System, Computing and Engineering (ICCSCE), Batu Ferringhi, 2016, pp. 441-445. doi: 10.1109/ICCSCE.2016.7893614

8. Willy Hereman and William S. Murphy, "Determination of a Position in Three Dimensions Using Trilateration and Approximate Distances" https://inside.mines.edu/ whereman/papers/Murphy-Hereman-TrilaterationMCS-07-1995.pdf [Online; accessed 2018-01-03]

9. Nan-Feng Xiao and S. Nahavandi, "Multi-agent model for robotic assembly system," Proceedings of the 5th Biannual World Automation Congress, Orlando, FL, 2002, pp. 495-500. doi: 10.1109/WAC.2002.1049486 